len, der Verbesserung der Umwelteigenschaften von Produkten und als Entscheidungsgrundlage für Beschaffung und Einkauf und kann schließlich Änderungen von Produktionsverfahren empfehlen. Nach unseren Erfahrungen kann eine Produkt-Ökobilanz dies zweifelsohne leisten, wenn sie sowohl für die entsprechende Vorproduktion als auch fuir die entsprechende Alternative vorliegt. Wir brauchen für unser Brot Mehl, Wasser und Salz - sonst nichts. Deswegen gelang dies in unserem Falle.

Wir setzen unsere Produkt-Ökobilanz zur Schwachstellenanalyse und vor allem im Kommunikationsbereich ein. In der PR-Arbeit wie auch in der Werbung müssen wir immer wieder darstellen, wo die Vorteile ökologischer Lebensmittel liegen und warum sie teurer sind. Dafür liefert die Produkt-Ökobilanz hervorragende Argumente. Beispielhaft haben wir dies in der Werbung zu der Aussage verdichtet: Ein PfisterBauernbrot schont 4 qm Bayern! Darüber hinaus dient die Ökobilanz als Bewerbungsunterlage für Umweltpreise und bildet schließlich die Basis für strategische Überlegungen, d. h. bei Produktentwicklung oder -änderungen. Die Verwendung der Produkt-Ökobilanz ist im Handel allerdings bisher auf wenig Gegenliebe gestoßen, denn in diesem Bereich dominiert das Preisargument!. Insgesamt dient die Produkt-Ökobilanz natïrlich der Imageverbesserung des Betriebes und zur Abgrenzung von Konkurrenzprodukten. Zusammenfassend lassen sich folgende Empfehlungen formulieren:

- Bevor man mit der Erstellung einer ProduktÖkobilanz beginnt, sollte man sich darüber im klaren sein, welches Ziel damit erreicht werden soll bzw. welchen Zweck man damit verfolgen will.

- Bei der Erstellung gilt es alle daran Beteiligten an der kurzen Leine zu führen, damit daraus kein „Jahrhundertwerk" wird.

- Nach der Fertigstellung sollte möglichst umgehend mit der zieladäquaten Umsetzung begonnen werden, denn manchmal schläft Ihr Mitwettbewerber nicht.

\section{Der Autor}

Friedbert Förster ist Diplom-Ökotrophologe und leitet die Marketingabteilung der Ludwig Stocker Hofpfisterei GmbH München.

Kontakt: Ludwig Stocker Hofpfisterei GmbH, Kreitlmayrstr. 5, 80335 München, Tel. 089/5202-0,

Fax 089/5202207.

Anwendungspraxis aus Sicht zweier Großunternehmen der Automobilindustrie

\title{
Interdisziplinärer Prozeß
}

\author{
Durch zahlreiche Bilanzierungsprojekte besitzt die Automobilindustrie breite \\ Erfahrungen mit Ökobilanzen. Dabei zeigt sich, daß diese Bilanzen ein zuneh* \\ mend brauchbares Instrument zur Informationsgewinnung über ökologische \\ Kosten sowie ökologische und ökonomische Verbesserungspotentiale werden \\ können - allerdings mit beträchtlichem Aufwand.
}

\section{I} Von Hans-Martin Beyer und Claudius Kaniut lanz-Aktivitäten zu Ende der achtziger Jahre (1). Sie mündeten in die Bildung des EUCAR-LCAArbeitskreises (EUCAR = European Council on Automobile Reserach; LCA = Life Cycle Assessment), einer Arbeitsgruppe von heute elf europäischen Automobilherstellern (2, 3). Beispiele für bereits durchgeführte bzw. laufende Ökobilanz-Studien im Automobilbereich betreffen zahlreiche Bauteile wie z. B. Stoßfänger, Kotflügel, Bodengruppe, Türen, Ölfilter, Benzintank, Achsteile, elektronische Komponenten und Lackierverfahren, ferner alternative Antriebe (z. B. alternative Kraftstoffe, Elektrotraktion), aber auch Standortentscheidungen (4).

Solche Bauteil- und zugehörige Verfahrensbilanzierungen sind Schritte auf dem Weg hin zur Bilanzierung vollständiger Automobile, die wegen ihrer außerordentlich großen Komplexität erst allmählich in Angriff genommen werden (5). Aus gleichem Grund beschränken sich Studien im Automobilbereich bisher vornehmlich auf Sachbilanzen. Die Wirkungsabschätzung findet aufgrund der international noch in Bewegung befindlichen Methodikentwicklung erst allmählich Berücksichtigung in Automobilbilanzierungen.

Um Ökobilanzen in Unternehmen zu initiieren und zu etablieren, bieten sich die im folgenden für den Automobilsektor skizzierten Bilanzierungsphasen an.

\section{- Bilanzierungsvorbereitung und -planung}

Voraussetzung für umweltrelevante Entwicklungen im Automobilbereich ist ein generelles Kundeninteresse an Ökothemen, das sich heute vor allem auf den Kraftstoffverbrauch und auf das Recycling konzentriert. Dagegen sind weitere Bemühungen der Automobilhersteller - wie $z$.
B. eine umweltgerechte Produktgestaltung - für den Laien nicht ohne weiteres erkennbar. Gerade hierfür aber können Ökobilanzen einen direkten Beitrag leisten. Da jedoch Kunden wie Medien den Grad des Bemühens um ganzheitlich umweltgerechte Produkte häufig nicht richtig einschätzen können, sind sie nur selten bereit, diesen nicht direkt „erlebbaren“ ökologischen Mehrwert zu honorieren. Dies kann zu einer zurückhaltenden Einstellung von Entscheidungsträgern hinsichtlich möglicher ÖkobilanzAktivitäten führen.

Da Entscheidungsträger immer nach dem Nutzen von Aktivitäten und dem „Return of Investment" fragen (müssen), ist es zunächst erforderlich, potentielle Vorzüge ökologischer Bilanzierungen für das eigene Unternehmen, aber auch für Zulieferer und deren Unterlieferanten herauszuarbeiten. Dabei muß sowohl dem heterogenen Kenntnisstand als auch den unterschiedlichen Interessen aller Involvierten Rechnung getragen werden. So haben Zulieferer oft die Befürchtung, daß durch die Offenlegung ihrer Ökodaten dem Kunden ,Automobilhersteller" gegenüber ein weiterer Kostendruck entstehen könnte. Hier gilt es, solchen Befürchtungen durch ,vertrauensbildende" Maßnahmen zu begegnen.

Wenn die grundsätzliche Zustimmung zur Durchfiihrung von Ökobilanzen auf einer möglichst hohen Managementebene erreicht ist, sind zunächst die konkreten Projektziele und der Bilanzierungsumfang festzulegen. Neben der Komplexität des Bilanzierungsobjekts sind diese abhängig von z. B. den bereitgestellten finanziellen Mitteln, dem verfügbaren Zeitbudget, dem Know-how und der Größe des Bilanzierungsteams, dem Reifegrad der Tools, der Zugriffsmöglichkeit auf Daten und der zugrundegelegten Methodik.

Immer wieder wird die Forderung nach „schnellen“, kostengiinstigen und trotzdem aus- 
sagefähigen Bilanzierungen gestellt. Sind umfassendes Ökobilanz-Know-how, anwendungsreife Tools, zahlreiche und qualitativ hochwertige Daten sowie eine ausreichende Anzahl von Bilanzierungsmodulen vorhanden, ist diese Forderung durchaus erfiillbar. Generell gilt jedoch das WYPIWYG-Prinzip (What You Pay Is What You Get), insbesondere bei „Erstbilanzierungen“, d. h. bei Produkten, die bisher nicht bilanziert wurden.

Aufgrund des großen Aufwandes für ,Erstbilanzierungen“ erscheint es zweckmäßig, das BasisKnow-how zunächst ,schnell“ mit externer Hilfe aufzubauen. Ist die notwendige Kernkompetenz erarbeitet, kann das unternehmensintern entstandene Bilanzierungswissen auch für vertrauliche, z. B. strategische Projekte eingesetzt werden und die externe Unterstützung reduziert werden.

\section{- Bilanzierungsdurchführung und -auswertung}

Die Datenakquisition dominiert mit durchschnittlich 80 Prozent des Gesamtaufwandes den Bilanzierungsprozeß. Vor allem in Großunternehmen ist sie besonders aufwendig, da Vielzahl und Vielschichtigkeit der Produkte sowie Anzahl und Unterschiedlichkeit betroffener interner und externer Stellen in der Bilanzierungsvorbereitung einen erheblichen Aufwand bedeuten. Hauptprobleme sind der unterschiedliche Kenntnisstand dieser Stellen und deren unterschiedliche Bereitschaft zur Mitwirkung. Solche Probleme gilt es zu lösen - durch eine hinreichende, rechtzeitige Motivierung und Heranfiihrung der Partner ans Thema.

Die Bilanzierungspraxis macht es häufig erforderlich, Daten unterschiedlicher Herkunft und Qualität zu verwenden. Dabei ist insbesondere auf Datensymmetrie und Transparenz zu achten. Aber auch die Allokation, d. h. die sinnvolle Zuordnung von Ökodaten zu bestimmten Leitgrößen, ist fallspezifisch abzuklären. Vorteilhaft im Hinblick auf Schnellbilanzierungen ist es auch, unabhängig von einem akuten Bilanzierungsbedarf „Schubladen-Bilanzierungen“ durchzuführen, um Daten und Bilanzierungsmodule kurzfristig verfügbar zu haben.

Von Interesse ist außerdem die Frage, ob Ökobilanzen durch ein zentrales Team oder dezentral durchgeführt werden sollen. Prinzipiell scheint die dezentrale Lösung in Großunternehmen vorteilhaft zu sein. Andererseits erfordern Komplexität und Vielfalt der Themen eine dauerhafte
Beschäftigung mit diesem Thema, um Expertenwissen und fundierte Aussagen zu erhalten. Ein zentraler Bilanzierungsbereich wird nach einer Phase des Know-how-Aufbaus in die Lage versetzt, zu einer unternehmens- und zuliefererübergreifenden einheitlichen Bilanzierungsmethodik und -durchführung, zu praktikablen Tools mit einheitlichen Ökodaten, etc. beizutragen und zusätzliche Aufgaben wie Bilanzierungssupport-koordination, Trouble-shooting, Knowhow-Transfer, Feedback- und Review-Aufgaben, etc. wahrzunehmen.

Auf der Basis der Bilanzierung bestehender Produkte und Prozesse können „Online-Bilanzierungen" in Angriff genommen werden, d. h. Bilanzierungen von Produkten, die sich in der Serienentwicklungsphase befinden und bei denen verschiedene Parameter nicht bzw. noch nicht endgïltig festgelegt sind. Hierdurch kann theoretisch die Produktgestaltung im positiven Sinne beeinflußt werden; in der Praxis sind viele Festlegungen aber bereits getroffen, so daß die gewonnenen Ergebnisse eher in Modellpflegemaßnahmen oder erst in Nachfolgeprodukten umgesetzt werden können.

Den größten Produktnutzen können Bilanzierungen erbringen, wenn sie bereits in der Planungs- oder Strategiephase neuer Produkte durchgefiuhrt werden. Da hier aber die meisten Festlegungen noch nicht getroffen wurden und zunächst mehrere Alternativen in Frage kommen, sind je nach Aufgabenstellung Alternativbilanzierungen, Szenariobetrachtungen, Best-/ Worst-Case-Studien, Sensitivitätsanalysen etc. durchzufuihren. Hierfür müssen ein umfassendes, langjähriges Bilanzierungs-Know-how, einsetzbare Bilanzierungstools und ein weitgefächerter hochwertiger Datenpool bereitstehen. Die Bilanzierungsauswertung erfolgt gemeinsam mit den unternehmensinternen Auftraggebern der Ökobilanz ggf. unter Einbeziehung externer Partner. Dabei werden Wirkungsabschätzung und Auswertung der Bilanzierung in Anlehnung an die Diskussion zur ISO 14042 und 14043 und in Abhängigkeit von der zugrundeliegenden Qualität der Sachbilanz sowie von produkt- wie unternehmensspezifischen Kriterien durchgeführt.

\section{- Handlungsempfehlungen}

Die Handlungsempfehlungen müssen zielgruppenorientiert erfolgen, indem z. B. Schwachstellen im Lebensweg herausgestellt werden, die ökologische und ökonomische Einsparpotentiale darstellen oder die zur Ableitung und Begründung von Technologieempfehlungen führen. Für die Umsetzung von Handlungsempfehlungen ist zu beachten, daß der Entscheidungsproze $ß$ in der Praxis interdisziplinär ist und die Ökobilanz nur ein Instrument in der gesamten ,Tool-box" der Entscheidungsfindung darstellt. Der Wunsch bzw. die Hoffnung mancher Ökobilanz-Verfechter, die Ökobilanz könne im industriellen (und politischen) Entscheidungsprozeß das maßgebende Instrument sein, ist daher unrealistisch. Selbstverständlich müssen Kosten-Nutzen-Aspekte wie auch unternehmensspezifische Kriterien (hinsichtlich Produktpalette, Komfort, Sicherheit, Qualität etc.) in den Entscheidungsfindungsprozeß einfließen. Die Umsetzung kann sich dabei je nach Aufgabenstellung auf die Stadien Konzeptphase, Gestaltungsphase, Serienentwicklung, Produktion, Nutzung und/oder Recycling/Entsorgung beziehen. Darüber hinaus ist auch grundsätzlich die Einbeziehung von LifeCycle-Costing-Aspekten vorstellbar.

\section{Aspekte bei internationalen Unternehmen}

Der Grad der Internationalisierung z. B. von Produktentwicklung, Einkauf und Marktanforderungen, aber auch der Sitz der Entscheidungszentrale hat großen Einfluß auf die Arbeit des Bilanzierungspraktikers. Die wichtigsten Aspekte, auf die im folgenden eingegangen wird, sind: höhere organisatorische bzw. strukturelle Komplexität, unterschiedliche nationale Problemgewichtung/-wahrnehmung, unterschiedliche Rahmenbedingungen durch nationale Gesetzgebung und internationale Unterschiede in der Akzeptanz der ÖkobilanzMethodik.

Komplexität: Internationale bzw. globale Unternehmen zeichnen sich im allgemeinen durch eine höhere organisatorische und strukturelle Komplexität aus, die sich zwangsläufig auf die Durchführung sowie die Kosten von Ökobilanzprojekten auswirkt. Beispiele können sein:

- Durch die Zahl und den Spezialisierungsgrad von betroffenen Bereichen bzw. Personen erhöht sich der Kommunikations- und Trainingsaufwand. - Kommunikationsprobleme internationaler Arbeitsgruppen potenzieren die spezifischen Probleme interdisziplinärer Arbeitsgruppen. 
Problemwahrnehmung: Nationale Unterschiede z. B. in der Bevölkerungsdichte, der ökologischen Tragfähigkeit, in der Umweltschädigung, aber auch in der Problemwahrnehmung der Bevölkerung können zu Anwendungsproblemen fiihren. Bereits in der Phase „Festlegung des Ziels und des Untersuchungsrahmens" können national bzw. regional unterschiedliche Problemperspektiven zu Schwierigkeiten bei der Konsensfindung über die Zielsetzung und die Bilanzgrenzen einer Studie führen. Dies gilt ebenso vor allem für die Auswertung zur Ermittlung des „ökologischen Optimums“. Hierbei können subjektive kulturelle Prioritäten wie auch politische bzw. legislative Prioritäten auf (supra)nationaler Ebene eine bedeutende Rolle spielen.

Rahmenbedingungen: Die jeweilige Umweltgesetzgebung hat mehr oder weniger direkten Einfluß auf die Ergebnisse der Sachbilanzierung. Beispiel hierfür sind national bzw. supranational gültige Schadstoff-Grenzwerte, die den Rahmen für Umweltbelastungen der Produktion bzw. Produktnutzung bilden. Internationale Unterschiede in gesetzlichen Regelungen führen im allgemeinen zur Erhöhung der Daten-Komplexität. Beispielhaft für den Automobilbereich ist die Anwendung unterschiedlicher „Drive cycles" zur Ermittlung des Fahrzeugverbrauches und der Fahrzeugemissionen zu nennen.

Im allgemeinen kann daher die These aufgestellt werden, daß eine Harmonisierung von gesetzlichen Regelungen die Sachbilanzierung vereinfachen würde. Wenig hilfreich sind Unterschiede in der Umweltgesetzgebung für die Bilanzbewertung bzw. -interpretation, da $z$. B. Grenzwerte nicht zwangsläufig mit den tatsächlich existierenden lokalen und regionalen Problemen korrelieren. Außerdem führen Unterschiede in der Umweltgesetzgebung zwangsläufig zu einer Einengung der Handlungsspielräume für ökobilanzgestützte Empfehlungen.

Von Bedeutung können aber auch unterschiedliche haftungsrechtliche Regelungen sein. Die hohen produkthaftungsrechtlichen Anforderungen beispielsweise in den USA führen zu erhöhter Besorgnis hinsichtlich der möglichen Konsequenzen von Entscheidungen, in die Ökobilanz-Resultate einfließen. Ökologische Empfehlungen, die zwangsläufig subjektive Wertungen hinsichtlich der relativen Bedeutung einzelner ökologischer Belastungen bzw.
Folgen enthalten, könnten als Fehleinschätzungen und daher haftungsrechtlich relevant interpretiert werden.

Akzeptanz: International ist die Akzeptanz der Ökobilanz-Methodik bislang unterschiedlich stark ausgeprägt. Derzeit ist sie in Europa am größten. Durch nationale Unterschiede können ohnehin existierende Problemfelder, z. B. die Bereitstellung finanzieller und personeller Ressourcen sowie die Bilanzplanung, -erstellung und praktische Umsetzung, zusätzlich verstärkt werden. Am bedeutendsten ist dabei ohne Zweifel der Rückhalt beim Management, der sich direkt auf die Bereitstellung von Mitteln sowie auf Entscheidungen zur Umsetzung von aus Ökobilanzen abgeleiteten Maßnahmenempfehlungen auswirkt.

Die Arbeit eines Bilanzierungspraktikers weist in internationalen Unternehmen natürlich nicht nur Probleme auf. Ganz im Gegenteil liegen gerade hier große Chancen, nämlich:

- Das für die Ökobilanzierung nötige Denken in großen Systemzusammenhängen unter Einschluß eines Verantwortungsgefühls für die Umweltprobleme auch außerhalb des eigenen, nationalen Rahmens sind naturgemäß mit dem Charakter eines internationalen Unternehmens voll kompatibel.

- Das Wissen um - wie auch das Verständnis für - die Umweltgesetzgebung anderer Nationen ist in international operierenden Unternehmen sehr groß, so daß die Arbeit des Praktikers erleichtert werden kann.

\section{Resỉmee}

Durch zahlreiche Bilanzierungsprojekte besitzt die Automobilindustrie zwischenzeitlich ein breites Erfahrungsspektrum mit Ökobilanzen. Es zeigt sich, daß diese Bilanzen mit zunehmender Erfahrung und wachsendem Datenbestand ein brauchund nutzbares Werkzeug zur Gewinnung ,objektiver" Informationen über ökologische Lasten, zur Erschließung von ökologischen wie ökonomischen Verbesserungspotentialen und damit für eine weiter umweltgerechtere Produktentwicklung - sowohl für kleine als auch für große Fahrzeuge - werden können.

Der Aufwand ist aber beträchtlich. Die Bereitschaft hierzu bei Unternehmen wird nur geweckt, wenn sich die Aufwendungen für Ökobilanzen durch pragmatische und anwendungsorientierte Lösungen in Grenzen halten und sich letztendlich auch amortisieren (z. B. durch Schwachstellen- analysen mit gezieltem Mitteleinsatz für Verbesserungen, durch ökologisch ,,richtige“ Produktentscheidungen, durch Vermeidung von Fehlinvestitionen, über Produktvergleiche und Benchmarks, durch Werbung, Imagegewinn etc.).

Gremien wie ISO, NAGUS, SETAC, SPOLD etc. können den Einsatz von Ökobilanzen im industriellen Umfeld durch praxisorientierte Methodenentwicklungen unterstïtzen. Aber auch wenn entsprechende Normen, ein ausreichender Datenbestand, anwendungsreife Tools und ein umfassendes Ökobilanz-Know-how verfügbar sein sollten, wird sich der Prozeß der Entscheidungsfindung in Großunternehmen auch künftig als ein interdisziplinärer Prozeß darstellen. Ökobilanzen können in diesem Entscheidungsfindungsprozeß eine wichtige Rolle spielen, sind aber eben ,nur" ein Instrument unter vielen (6).

\section{Liferafur}

(1) K. Bulitta: Stand der Ökobilanzierung in der europäischen Automobilindustrie. Interner Bericht Ford-Werke, Köln 1994

(2) Kaniut, C.; Beyer, H.M.; Neumann, U.: ÖkobilanzGemeinschaftsprojekt („EUCAR-LCA“) europäischer Automobilhersteller, Utech Berlin, Tagung Produktbezogene Ökobjlanzen IV, 29.2.-1.3.1996, Tagungsband, S. 99 - 117 (3) Beyer, H.-M.: Life Cycle Assessment - Combined Efforts of the European Automotive Industry, SAE Conference Detroit 7.-9.4.1997, Proceedings of the 1997 Total Life Cycle Conference - Life Cycle Management and Assessment (Part 1), SAE Paper 971172 , p. $97-106$

(4) Beyer, H.M.: Ökobilanz - Eine Methodik für Standortentscheidungen?, Tagung "Ganzheitliche Betrachtungen im Automobilbau", Wolfsburg 27.-29.11.1996, VDI-Berichte 1307, S. $373-393$

(5) Kaniut, C.; Cetiner, H.; Franzeck, J.: Life Cycle Assessment of a Complete Car - The Mercedes-Benz Approach, SAE Conference Detroit 7.-9.4.1997, Proceedings of the 1997 Total Life Cycle Conference - Life Cycle Management and Assessment (Part 1), SAE Paper 971166, p. 55 - 62

(6) Kaniut, C.; Cetiner, H.; Siehler, U.; Diener, J.: Ökologische Bilanzierung im Automobilbau am Beispiel von Verbundbauteilen, DGM-Tagung Kaiserslautern 17.-19.9.1997, Tagungsband, S. 55 - 65

\section{Die Autoren}

Dr. Hans-Martin Beyer ist Mitarbeiter der Ford-Werke AG, Direktiion Umwelischutz und Sicherheil, zuständig für Strategie und Geschäfisplanung. Dr.-Ing. Claudius Kaniut ist Mitarbeiter der Daimler-Benz AG, PKWVorentwicklung, Leiter Ganzheitliche Bilanzierung Kontakt: Ford-Werke AG, NG/OF-6, 50725 Köln, Fax 0221/9012610; Daimler Benz AG, EP/VUG T724, 70546 Stutigart, Fax 0711/1747-068 
(c) 20I0 Authors; licensee IÖW and oekom verlag. This is an article distributed under the terms of the Creative Commons Attribution Non-Commercial No Derivates License (http://creativecommons.org/licenses/by-nc-nd/3.o/), which permits unrestricted use, distribution, and reproduction in any medium, provided the original work is properly cited. 